\title{
IN THIS ISSUE
}

\section{Mosaic cells can muddy NIPS results}

\section{see page 620}

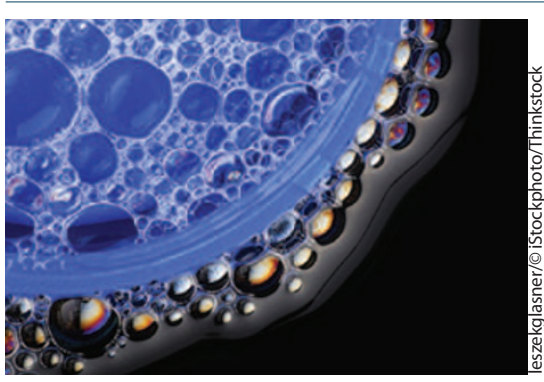

A recent retrospective study of nearly 53,000 prenatal tests for common fetal chromosomal abnormalities underscores the need for clarity when offering noninvasive prenatal screening (NIPS) to pregnant women. Grati et al. found that fetoplacental mosaicism, in which placental and fetal cells differ in containing aneuploid cells, can result in small but measurable rates of both false-positive and false-negative findings. The study, which analyzed the results from a large database of prenatal testing cases in which NIPS results were confirmed by direct testing of placental and fetal DNA, showed that for the common trisomies $(13,18$, and 21) false-positives can be expected in 1 in 3,006 cases and false-negatives can be expected in 1 in 107. The potential for false results led the authors to suggest that those offering NIPS counsel patients that the tissue being sampled is of placental origin and thus may not perfectly reflect the status of the fetus. To ensure that patients understand the implications of fetal-placental mosaicism, the authors propose that the term "cell-free fetal DNA" be changed to "cell-free placental DNA." The change in terminology should help providers inform patients of the test's limitations and the potential need for confirmatory invasive testing. - Karyn Hede, News Editor

\section{NIPT extended to diagnosis of single-gene recessive disorder}

\section{see page 594}

A Chinese research team has applied a combination of massively parallel sequencing and noninvasive prenatal testing to diagnose a single-gene autosomal recessive disorder within a clinically actionable time frame. The report presents a

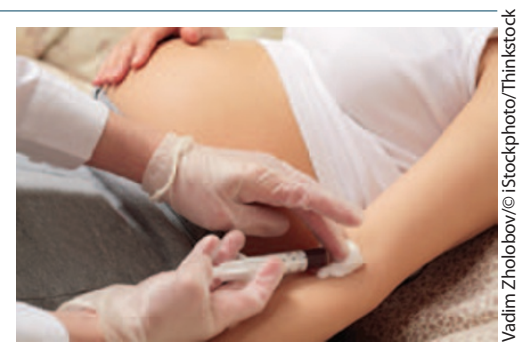
proof-of-principle clinical case showing the potential for noninvasive prenatal testing (NIPT) to be useful in diagnosing Mendelian disorders in the prenatal setting. In this case, the researchers worked with parents known to be carriers of maple syrup urine disease (MSUD), an autosomal recessive metabolic disorder with at least 40 known genetic variants in four genes that constitute an enzyme complex essential for breaking down branched-chain amino acids. The family had one infant diagnosed with MSUD, and the mother was pregnant again. Sequencing revealed a shared exon duplication in the affected child and the father, as well as a mutation, c.392A $>\mathrm{G}$, in the BCKDHA gene in the affected child and the mother. The novel missense mutation was expected to be deleterious, in part because it appeared in a highly evolutionarily conserved region of the gene. Prenatal NIPT revealed that the fetus had inherited the same defective alleles that the affected child had. The researchers confirmed the findings with amniocentesis. The testing took 50 days, with a definitive diagnosis made in the twentieth week of pregnancy. - Karyn Hede, News Editor

\section{NEWS BRIEFS}

\section{Tracking the DNA signatures of foodborne pathogens}

US government agencies are now cooperating to track down the source of foodborne contamination using wholegenome sequencing (WGS) of the contaminant bacteria. In March 2014, the US Food and Drug Administration (FDA) identified a strain of Listeria responsible for a multistate food poisoning outbreak and linked it to a factory producing cheese products. The DNA evidence supported an FDA decision to suspend production at the facility. It was the first time that the FDA and the Centers for Disease Control and Prevention (CDC) had worked together to connect envi-

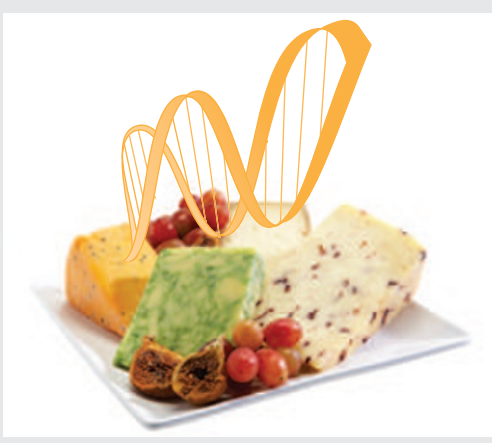

ronmental and food samples with the $C D C$ 's samples derived from humans. In addition, the FDA is working with public health agencies worldwide to sequence disease-causing bacteria collected from foodborne outbreaks. Sequencing data will be available in a public database called GenomeTrakr, which will be used to track the spread of contaminated food and to contain outbreaks. More than 5,000 Salmonella, Listeria, and Escherichia coli genomes have been added to GenomeTrakr; more are being added from samples collected at foodproduction facilities during inspections. Future plans call for working with the World Health Organization to expand WGS to food-safety laboratories worldwide. Italian food-safety experts have already received WGS training, according to an FDA consumer update, and the agency reports that it is planning a trip to Ireland to work with its food-safetyagency counterpart.

-Karyn Hede, News Editor 


\section{NEWS BRIEFS (continued)}

\section{Cloud storage: not just for your music collection}

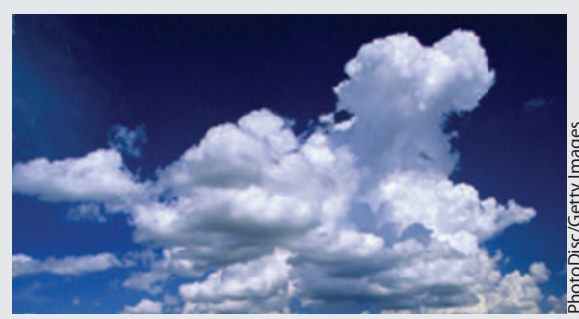

Search engine giant Google has signed its first deal to store genomic data, after announcing in February 2014 that it had built an interface specifically for genomic da- tasets. The advocacy organization Autism Speaks is teaming with Google to store the data generated through its Ten Thousand Genomes Program, which aims to use genomic information to unravel the genomic contribution to autism spectrum disorders. The organization is working with researchers in China to collect and sequence DNA samples. After reaching 1,000 genomes, according to the organization's scientific director, the group was quickly exhausting its data-storage capacity. Google's cloud storage and analytical tools seem well suited to expand the organization's storage and analytic capabilities. Autism Speaks has said that the project's private data will be accessible to researchers worldwide, but the terms of the deal are not publicly available, raising interesting and potentially thorny questions about long-term accessibility. Now that Google has fully entered the business of managing genomic data, it may be time to look at the fine print in that end-user agreement. What if Google, or another company offering private data storage, decides to back out of the agreement in the future? If publicly funded research depends on continued access to data controlled by a private, forprofit entity, who controls the data? The legal issues related to cloud computing are in flux and could affect genomic research well into the future.

-Karyn Hede, News Editor

\section{Genetics in Medicine | Mission Statement}

Genetics in Medicine is a monthly journal committed to the timely publication of:

- Original reports which enhance the knowledge and practice of medical genetics

- Strategies and innovative approaches to the education of medical providers at all levels in the realm of genetics

As the official journal of the American College of Medical Genetics and Genomics (ACMG), the journal will:

- Provide a forum for discussion, debate and innovation concerning the changing and expanding role of medical genetics within the broader context of medicine

- Fulfill our responsibility to the College membership through the publication of guidelines, policy statements and other information that enhances the practice and understanding of medical genetics

Finally, as genetics becomes increasingly important in the wider medical arena, we will be an accessible and authoritative resource for the dissemination of medical genetic knowledge to providers outside of the genetics community through appropriate reviews, discussions, recommendations and guidelines. 